\title{
Minimally invasive aortic valve reconstruction with autologous pericardium: how we do it
}

\author{
Dinh H. Nguyen ${ }^{1,2}$, Anh T. Vo ${ }^{2}$, Trang T. Nguyen ${ }^{2}$, Khoi M. Le ${ }^{2}$, Binh Q. Truong ${ }^{3}$, Bac H. Nguyen ${ }^{4}$ \\ ${ }^{1}$ Department of Cardiothoracic and Vascular Surgery, ${ }^{2}$ Department of Cardiovascular Surgery, University Medical Center, ${ }^{3}$ Department of \\ Interventional Cardiology, University Medical Center, ${ }^{4}$ Department of Surgery, University of Medicine and Pharmacy, Ho Chi Minh City, Vietnam \\ Correspondence to: Dinh H. Nguyen, MD. 16.07 LP2 Lucky Palace apartment, Ward 2, District 6, Ho Chi Minh City, Vietnam. \\ Email: tuananh21285@yahoo.com.
}

Submitted Jan 18, 2019. Accepted for publication May 03, 2019.

doi: 10.21037/acs.2019.05.12

View this article at: http://dx.doi.org/10.21037/acs.2019.05.12

\section{Clinical vignette}

A 22-year-old male was admitted with dyspnea on exertion due to a congenital aortic valve disease. Preoperative transthoracic echocardiography showed a severe aortic regurgitation and a quadricuspid aortic valve. The fourth cusp was aplastic, thus create a gap between the left and the non-coronary cusp, resulting in a regurgitant jet through this discontinuity. Echocardiographic examination also revealed a decreased left ventricular ejection fraction (LVEF) of $47 \%$, left ventricular end systolic diameter was $52 \mathrm{~mm}$. The diameter of the aortic annulus, sinotubular junction and ascending aorta were $30.8,33.4$ and $32.6 \mathrm{~mm}$, respectively. Right ventricle contractility was normal with a tricuspid annular plane systolic excursion (TAPSE) of $22 \mathrm{~mm}$ and the fractional area change (FAC) of $50 \%$. The vena contracta of the regurgitation was $6.8 \mathrm{~mm}$. The patient was in New York Heart Association (NYHA) Functional Class II. On the other hand, his medical history was unremarkable.

\section{Surgical techniques}

\section{Preparation and exposition}

The patient was placed in the supine position with a cushion under the back to facilitate exposure of the ascending aorta and aortic root. A single lumen endotracheal tube intubation was used. We did not routinely apply single lung ventilation.

\section{Operation}

The pericardium can be harvested in two different manners: endoscopic harvesting and direct harvesting. If the surgeon chooses the 4th intercostal space for the upper ministernotomy, direct harvesting through this approach can be performed. However, when one becomes more comfortable doing the procedure, the 3 rd intercostal space could be chosen for the ministernomy; at this level, thoracic endoscopy would provide an excellent exposure of the pericardium and make the harvesting achievable.

The thymus remnant and fat tissue was completely removed and the pericardium was transversely opened at its reflection. The pericardiotomy is then continued to the right via the superior vena cava, care should be taken not to damage the right phrenic nerve in this region. A wet $4 \mathrm{~cm} \times 4 \mathrm{~cm}$ gauze is then used to bluntly dissect the left pleura out of the pericardium. This dissection should go as far as possible to facilitate the subsequent pericardial harvesting and shorten the cardiopulmonary bypass $(\mathrm{CPB})$ time during this phase. $\mathrm{CPB}$ is established with femoral vein and ascending aorta cannulation.

\section{Direct pericardial harvesting}

After the CPB is initiated, the heart is emptied and the lungs are deflated, the pericardiotomy is then continued from the right toward the right diaphragm. Surgical clips are used to hold and pull up the pericardium for harvesting. A wet $4 \mathrm{~cm} \times 4 \mathrm{~cm}$ gauze is put under the right atrium to protect this structure from the electrocautery. When the right diaphragm is reached, the pericardiotomy is then changed to the left via the apex. An army navy retractor could be used to lift up the sternum to provide a better exposure. Traction sutures can also be used to pull up the 
pericardium. A $4 \times 4$ gauze is put between the heart and the pericardium for safety reason. When moving toward the apex, the surgeon need to recognise clearly the left anterior descending (LAD) artery in order to avoid damaging this structure. After reaching the apex, the pericardiotomy is continued cranially toward the left atrial appendage. The left pleural space is opened to identify the left phrenic nerve. Once the atrial appendage is reached, the surgeon can complete the harvesting by connecting this point with the beginning point.

\section{Endoscopic harvesting}

After CPB is initiated, one $10 \mathrm{~mm}$ and one $5 \mathrm{~mm}$ endoscopic trocars are placed in the 4th and 6th intercostal space at the level of the anterior axillary line and a $10-\mathrm{mm}$ endoscopic trocar in the 5th intercostal space at the level of the middle axillary line. The small port serves as a camera port for the endoscopic harvesting. The pericardiotomy is then continued parallel and $2 \mathrm{~cm}$ away from the phrenic nerve to the diaphragm and then curved up perpendicularly to go toward the apex. The fat pad at the cardiophrenic angle should also be completely removed to better expose the pericardium. A traction suture is placed on the edge of the pericardial patch and taken out through the ministernotomy to provide better exposure and to stretch the pericardium. One should place a wet gauge between the heart and the pericardium to protect the heart from the electrocautery. The pericardiotomy was continued until the apex and then curves up via the left atrial appendage. After reaching the reflection at the level of the pulmonary trunk, the pericardial patch was taken out via the ministernotomy.

The redundant fat tissue on the outer surface of the pericardium is removed, the caudal part is marked for subsequent template drawing, the bigger cusp was placed at the in this region. The patch is treated with glutaraldehyde $0.6 \%$ with a buffer for 10 minutes and rinsed 3 times with normal saline, each time 6 minutes.

Cardioplegia is directly given via the coronary ostia. Aortic cusps are removed carefully. The interval between the commissures are measured using a specially designed sizer set (AVNeo Sizer, Nihonbashi Life Science, Tokyo, Japan). Appropriate sizing is an important key in this procedure, proper tension should be applied on the annulus, similar to the annular diastolic pressure. The corresponding size of each cusp is marked and the new pericardial cusps are trimmed conformable to an original template which is also available with the sizers in the instrument set.

The newly created cusps had two borders, the annular border and the free border. The annular border was sutured to the native annular. At the nadir of the annulus, the ratio of the interval between each bite on the patch and the aortic annulus was $3: 1$. This proportion would be changed to $1: 1$ at the commissural area. An additional 4-0 Prolene was placed at the extension wings at each commissure to reinforce this area. The coaptation of the new valve was then checked visually and by putting a wall suction into the left ventricle to see these leaflets collapse.

\section{Completion}

The aortotomy is closed, the heart de-aired and after cross-clamp removal, postprocedural transesophageal echocardiography (TEE) is critical to assess valve function. A pre-discharge TTE is performed on all patients and again at 1,3 and 6 months postoperatively.

\section{Comments}

Before Ozaki, many authors have reported the usage of autologous pericardium in repairing or replacing the aortic valve (1-3) and emphasized the benefit of direct suturing of the pericardial leaflet to the native annulus. Nevertheless, the mid-term and long-term results of the technique in younger populations remains unclear. Liu et al. have published their long-term results in aortic valve replacements using autologous pericardium in 15 young patients (4), with a mean age of $34 \pm 12$ years and mean follow-up time of $11.43 \pm 4.50$ years. The reoperation rate was fairly acceptable at $33 \%$ (five patients). Jeong et al. in 2009 have also reported the long-term results of the leaflet extension technique in aortic valve repair using autologous pericardium (5). Ozaki et al. has also reported promising midterm results of their cohort $(6,7)$. Regarding the approach, current evidence suggests that the ministernotomy approach for aortic valve operation is associated with many advantages: shorter ventilation time, shorter ICU time and hospital stay and less blood loss (8). Its safety and efficacy are as good as the conventional approach via the full sternotomy (9).

In 1.5 years, nine patients with severe aortic valve diseases underwent minimally invasive aortic valve reconstruction with autologous pericardium through an upper ministernotomy approach at the University Medical Center of Ho Chi Minh City, Vietnam. The mean age was $47.4 \%$ and $55.6 \%$ were female. The average NYHA class was 1.6. Most patients had good ventricular function with a mean LVEF of $62.3 \%$. The dominant pathology was post-rheumatic valve disease 
(6/9 patients, 66.7\%) and three patients were diagnosed with bicuspid aortic valve disease. Five patients had severe aortic regurgitation, three had severe aortic stenosis and one had a combined stenosis and regurgitation valve.

No in hospital and 30-day mortality was recorded in our series and no conversion to full sternotomy was required. Mean cross clamped time was 106.8 minutes, mean CPB was 153.6 minutes, mean ventilation time was 8.4 hours and mean ICU time was 1.6 days. The CPB time for endoscopic pericardial harvesting reduced gradually case after case and may shorten even more in the future.

One patient needed to change the valve due to more than moderate regurgitation on TEE. This was a bicuspid aortic valve which also required neocommissural reconstruction. One patient developed a right hemothorax requiring drainage at postoperative day 1 .

A minimally invasive approach combined with an Ozaki procedure is feasible, safe and is an alternative to conventional sternotomy surgery. The latter should be reserved for severe patients. In our center, this approach is associated with low mortality and morbidity and a high successful rate of repair. However, further assessments are required to make a consolidate conclusion.

\section{Acknowledgments}

None.

\section{Footnote}

Conflicts of Interest: The authors have no conflicts of interest to declare.

\section{References}

1. Grabenwöger M, Fitzal F, Gross C, et al. Different modes

Cite this article as: Nguyen DH, Vo AT, Nguyen TT, Le KM, Truong BQ, Nguyen BH. Minimally invasive aortic valve reconstruction with autologous pericardium: how we do it. Ann Cardiothorac Surg 2019;8(3):444-446. doi: 10.21037/ acs.2019.05.12 of degeneration in autologous and heterologous heart valve prostheses. J Heart Valve Dis 2000;9:104-9; discussion 110-1.

2. Duran CM, Gometza B, Kumar N, et al. Aortic valve replacement with freehand autologous pericardium. J Thorac Cardiovasc Surg 1995;110:511-6.

3. Aupart MR, Sirinelli AL, Diemont FF, et al. The last generation of pericardial valves in the aortic position: ten-year follow-up in 589 patients. Ann Thorac Surg 1996;61:615-20.

4. Liu X, Han L, Song Z, et al. Aortic valve replacement with autologous pericardium: long-term follow-up of 15 patients and in vivo histopathological changes of autologous pericardium. Interact Cardiovasc Thorac Surg 2013;16:123-8.

5. Jeong DS, Kim KH, Ahn H. Long-term results of the leaflet extension technique in aortic regurgitation: thirteen years of experience in a single center. Ann Thorac Surg 2009;88:83-9.

6. Ozaki S, Kawase I, Yamashita H, et al. A total of 404 cases of aortic valve reconstruction with glutaraldehydetreated autologous pericardium. J Thorac Cardiovasc Surg 2014;147:301-6.

7. Ozaki S, Kawase I, Yamashita H, et al. Midterm outcomes after aortic valve neocuspidization with glutaraldehydetreated autologous pericardium. J Thorac Cardiovasc Surg 2018;155:2379-87.

8. Brown ML, McKellar SH, Sundt TM, et al. Ministernotomy versus conventional sternotomy for aortic valve replacement: a systematic review and meta-analysis. J Thorac Cardiovasc Surg 2009;137:670-9.e5.

9. Dalén M, Biancari F, Rubino AS, et al. Ministernotomy versus full sternotomy aortic valve replacement with a sutureless bioprosthesis: a multicenter study. Ann Thorac Surg 2015;99:524-30. 